\title{
Hypoxia preconditioning promotes mesenchymal stem cells survival and vascularization through the activation of MALAT1/miR-195/VEGFA axis
}

Jingying Hou ( $\square$ houjy6@mail.sysu.edu.cn )

sun yat sen memorial hospital of sun yat sen university

Lingyun Wang

sun yat sen memorial hospital of sun yat sen university

Zhengfei Yang

sun yat sen memorial hospital of sun yat sen university

Guanghui Zheng

sun yat sen memorial hospital of sun yat sen university

Tianzhu Guo

sun yat sen memorial hospital of sun yat sen university

Menglei Yu

sun yat sen memorial hospital of sun yat sen university

\section{Research}

Keywords: Hypoxia preconditioning; mesenchymal stem cells; survival; vascularization; long noncoding RNA MALAT1; microRNA-195; vascular endothelial growth factor A

Posted Date: March 12th, 2020

DOl: https://doi.org/10.21203/rs.3.rs-17014/v1

License: (c) (i) This work is licensed under a Creative Commons Attribution 4.0 International License.

Read Full License 


\section{Abstract}

Background: Previous studies have demonstrated that hypoxia preconditioning (HP) can promote mesenchymal stem cells (MSCs) survival and vascularization. Long noncoding RNA metastasisassociated lung adenocarcinoma transcript 1 (MALAT1) is a newly discovered regulator of MSCs viability and differentiation. Evidences have indicated that MALAT1 can be strongly induced by hypoxia. This study aimed to investigate the role of MALAT1 in HP mediated MSCs survival and vascularization as well as the relevant underlying mechanism in vitro.

Methods: MSCs were obtained from C57BL/ 6 mice and cultured in vitro. Cells at the third passage were divided into the following groups: normoxia (N), hypoxia preconditioning (HP), HP + MALAT1, HP + MALAT1 NC, HP+si-MALAT1 and HP +si-MALAT1 NC. The normoxia group was cultured in $20 \% 02$ for 24 h. All the other groups were exposed to hypoxia (1\% 02) for 24 hours. MALAT1 and relevant scramble RNA were transfected in the HP+MALAT1 and HP+MALAT1 NC groups respectively. HP+si-MALAT1 and HP +si-MALAT1 NC groups were transfected with MALAT1 siRNA and relevant siRNA scramble respectively. MSCs proliferation, apoptosis and vascular densities were evaluated. Bioinformatics and dual luciferase reporter assay were performed. Relevant biomarkers were examined in different experimental groups.

Results: MSCs survival and vascularization were significantly enhanced in the HP group. Transfection of MALAT1 further strengthened the viability and angiogenic potential of MSCs in the condition of HP, whereas its knockdown attenuated cells survival and vascularization. MALAT1 and vascular endothelial growth factor A (VEGFA) were obviously increased after hypoxia exposure, while miR-195 was decreased. miR-195 targeted and downregulated VEGFA. miR-195 was a target of MALAT1. Overexpression of MALAT1 led to a decreased level of miR-195, accompanied with an augmented expression of VEGFA. However, both miR-195 and VEGFA exhibited contrary alterations after MALAT1 blockage.

Conclusion: HP enhanced MSCs survival and vascularization potential in vitro, and the activation of MALAT1/miR-195/VEGFA axis might be involved in this procedure. This study reveals a new molecular mechanism of HP mediated MSCs survival and vascularization. It will be conducive for the development of novel strategies to improve the therapeutic efficiency of MSCs based on HP.

\section{Introduction}

Mesenchymal stem cells (MSCs) therapy is widely used in the area of cardiovascular regeneration [1]. Plenty of animal and human studies have certified that MSCs transplantation can repair the injured heart and boost cardiac function [2, 3]. However, their therapeutic efficacy remains unsatisfactory. One major constraint is the poor survival and fragile angiogenic capability of the implanted cells after injection [4]. In consideration of this, it is urgently in need to probe into the determinants that manipulate MSCs survival and vascularization. Diversified approaches have been applied to improve the status of these cells in order to further ameliorate their therapeutic efficacy [5]. Utilization of hypoxia preconditioning (HP) has 
been accredited as an available strategy to optimize MSCs survival and functional capacity [6, 7]. Evidences show that both the viability and angiogenic potential of MSCs are notably reinforced after HP [8]. MSCs experienced HP exhibits a greater therapeutic advantage in the hostile ischemic microenvironment in vivo $[8,9]$. Nevertheless, the intricate mechanism of HP mediated effects is still poorly understood.

Recent studies reveal that long noncoding RNAs (IncRNAs) can act as regulators of stem cells biological activities, including stemness maintenance, cells proliferation and differentiation [10-12]. Additionally, they are also closely associated with vascular function and angiogenesis [12]. IncRNA metastasis associated lung adenocarcinoma transcript 1 (MALAT1), which is located within human chromosome $11 \mathrm{q} 13$ and mouse chromosome 19qA, was originally identified as a prognostic parameter for metastasis and patient survival in non-small cell lung cancer (NSCLC). [13]. It is abundantly expressed in a variety of tissues and regulates the expression of genes entangled with proliferation and cellular motility [14]. Previous data have displayed that MALAT1 is engaged in MSCs proliferation and vascularization [15].

Hypoxia upregulates a series of genes involved in cell migration, apoptosis, angiogenesis and stemness [16-18]. Several studies indicate that hypoxia escalates the expression level of MALAT1 [16, 17]. However, it remains unclear whether MALAT1 is involved in HP mediated MSCs survival and vascularization and what might be the specific mechanism. In this study, we tried to investigate the role of MALAT1 in HP mediated MSCs survival and vascularization in vitro and explored the relevant mechanism.

\section{Materials And Methods}

\section{Ethics Statement}

3 weeks old C57BL/ 6 mice were obtained from the Animal Experimental Center of the Sun Yat-sen University. All animal handling and procedures were performed in accordance with protocols approved by the Animal Ethics Committee of Sun Yat-sen University (201900005).

\section{Isolation and Culture of MSCs}

Isolation, culture and identification of MSCs were performed as previously reported $[12,19,20]$. The third passage MSCs were used for all the experiments.

\section{Plasmid construction}

For the overexpression of MALAT1, pcDNA3.1 vector containing the sequence of MALAT1 was applied for the amplification of MALAT1 (Youbio, Cat: G30218). The primers were as follows: Xho1 forward: 5'ctcgag CAGGCATTCAGGCAGCGAGA-3', Pmel reverse: 5'-gtttaaac TGAGAGATATTTAGTTTTTA-3'. Plasmid carrying a non-targeting sequence was used as a negative control. For the knockdown of MALAT1, three complementary oligonucleotides of siRNA, MALAT1-siRNA1\# (5'-AAGACAACGGAGCCAGCAdTdT-3'), MALAT1-siRNA2\# (5'-gaagagaggaggagcagcdTdT-3') and MALAT1-siRNA3\# (5'- 
acagggcgggccggTGGGdTdT-3') and a scramble negative siRNA (5'-GAACUGGGGUGCGUGUGAUdTdT-3') were constructed.

\section{Transient transfection experiments}

Transfection of MSCs was performed by using Lipofectamine2000 (Invitrogen) as previously [12]. MSCs were incubated at $1 \times 10^{6}$ cells per well in six-well plates. MALAT1, MALAT1 NC, MALAT1 siRNA, siMALAT1 NC, miR-195 mimic, miR-195 NC, miR-195 inhibitor and miR-195 inhibitor NC were transiently transfected into MSCs. Overexpression or knockdown of MALAT1 and miR-195 was verified by quantitative real-time PCR.

\section{Hypoxia preconditioning treatment of MSCs}

MSCs in different experimental groups were incubated in $1 \% \mathrm{O}_{2}$ in Galaxy ${ }^{\circledR} 48 \mathrm{R}$ incubator (Eppendorf/Galaxy Corporation, USA) at $37{ }^{\circ} \mathrm{C}$ for $24 \mathrm{~h}$. Normoxia $\left(20 \% \mathrm{O}_{2}\right)$ was used as a blank control during the whole process for all the groups $[12,19]$.

\section{Evaluation of MSCs proliferation and apoptosis}

MSCs of different groups were collected and suspended in complete culture medium. The MTS assay (cellTiter96AQ, one solution cell proliferation assay, catalogue number G3582; Promega, Madison, Wisconsin, USA) was applied to evaluate cells proliferation as previously [12, 19]. MSCs apoptosis was estimated by the terminal deoxynucleotidyl transferase biotin-dUPT nick end-labeling (TUNEL) assay as previously $[12,19]$. All sections were examined and results were calculated under a florescent microscope (DMI6000B; Leica, Brunswick, Germany) [12, 19].

\section{Vascular tube formation assay}

Aliquots of human umbilical vein endothelial cells (HUVECs, Yiyuan biotechnology corporation, GuangZhou, China) were seeded onto matrigel-coated wells (BD corporation, Cat.No. 356234) of a 24well plate. Vascular tube formation assay was performed as previously $[12,19]$. The numbers of the vascular branches were detected by using a phase-contrast microscopy (OLYMPUS CKX41, U-CTR30-2, Japan) as previously described $[12,19]$.

\section{Vector construction and luciferase reporter assay}

Two luciferase reporters containing the wild type MALAT1 (psiCHECK2-MALAT1-WT) or mutant MALAT1 were constructed to validate the interaction between MALAT1and miR-195. MALAT1 (containing the binding sites for miR-195) was expanded with the following primer sequences: forward (F): 5'AATTCTAGGCGATCGCTCGAGCAGGCATTCAGGCAGCGAGA-3' and reverse (R): 5'GCGGCCGCTCTAGGTTTAAACTGAGAGATATTTAGTTTTTATTTCATA- AAATC-3'. Mutant MALAT1 contained a mutation site (psiCHECK2-MALAT1-MU) which eliminated the miR-195 targeting, and its primer sequences were as follows: MALAT1-mut (2427 bp-2447 bp): F: 5'-

TGGGTTAGAGAAGGCGTTCGCCGACACTACTCCGGCACGACACCT-TCAGG-3', R: 5'CCTGAAGGTGTCGTGCCGGAGTAGTGTCGGCGAACGCCTTCTCTAACCCA-3'; MALAT1-mut (5013 bp- 
5038 bp): F: 5'-TGGCAAGTAATATGTGAGTCGCCTCCACAATACTACTACATTAGAAT-GCATTGTGA-3', R: 5'TCACAATGCATTCTAATGTAGTAGTATTGTGGAGGCGACTCACATATTACTTGCCA-3'; MALAT1-mut (5201 bp-5221 bp): F: 5'-GTGATTGGAGCCGAGTCCCTCCGACTCTACTACTTAGGTAAATGTTT-3', R: 5'AAAACATTTACCTAAGTAGTAGAGTCGGAGGGACTCGGCTCCAATCAC-3'. Lu--ciferase reporter assay was performed with the dual-luciferase reporter assay system (Promega) and the relative luciferase activity was calculated as previously [12].

\section{Western blot analysis}

Protein levels were assessed by western blot as previously reported $[12,19,20]$. The bands were examined by enhanced chemiluminescence after extensive washing, and quantitive analysis of bands intensities was performed by using image software (image $\mathrm{J} 2 \mathrm{x}$, version 2.1.4.7) $[12,19,20]$.

\section{Quantitative real-time polymerase chain reaction (qRT-PCR) analysis}

Quantitative real-time polymerase chain reaction (qRT-PCR) was performed on a ABI PRISM ${ }^{\circledR} 7500$ Sequence Detection System by using SYBR Green qPCR SuperMix (Invitrogen) as previously $[12,19,20]$.. The primers were described in Table 1. Specific products were amplified and examined with Applied Biosystems at $95^{\circ} \mathrm{C}$ for $10 \mathrm{~min}$, followed by 40 cycles at $95^{\circ} \mathrm{C}$ for $15 \mathrm{~s}$ and at $60^{\circ} \mathrm{C}$ for $30 \mathrm{~s}$, at which point data were acquired. The $2^{-\Delta \Delta C t}$ method was used for the calculation of the relative level of mRNA as previously $[12,19,20]$.

Table 1

List of primers for qRT-PCR

\begin{tabular}{|lll|}
\hline Gene name & Forward primer $\left(\mathbf{5}^{\prime} \mathbf{3}^{\prime} \mathbf{)}\right.$ & Reverse primer $\left(\mathbf{5}^{\prime} \mathbf{3}^{\prime} \mathbf{)}\right.$ \\
\hline MALAT1 & GAAGATAGGCATTTGAGTGGCT & CTGAAGAGCATTGGAGATCAGC \\
VEGFA & ATGCCGGTTCCAACCAGAA & GTGGAGGAGCGAGCTGAA \\
miR-195 & ACACTCCAGCTGGGCCCAGTGTTCAGACTACCT & CTCAACTGGTGTCGTGGA \\
\hline U6 & CTCGCTTCGGCAGCACA & AACGCTTCACGAATTTGCGT \\
\hline GAPDH & tggccgtggggctgcccag & ggaaggccatgccagtgagc \\
\hline
\end{tabular}

\section{Statistic analysis}

All quantitative data were described as mean \pm SD. The significance of differences among groups was determined by analysis of variance and Scheffe's multiple-comparison techniques. Comparisons between time-based measurements within each group were performed with analysis of variance for repeated measurements. A value of $p<0.05$ was considered to be statistically significant.

\section{Results}




\section{Interference efficiency of MALAT1 siRNA and the expression of MALAT1 in different groups}

The mRNA level of MALAT1 was examined in order to evaluate of the interference efficiency of MALAT1 siRNA. MALAT1 expression status was confirmed by qRT-PCR analysis. It was revealed that the MALAT1siRNA1\# with the concentration of $100 \mathrm{nM}$ exhibited the highest inhibition efficiency and was used in the subsequent experiments $(P<0.01$; Fig. 1A). The mRNA level of MALAT1 was distinctly increased in the HP + MALAT1 group compared with HP + MALAT1 NC and HP groups, while its expression level was significantly decreased in the HP + si- MALAT1 group in contrast with the HP + si-MALAT1 NC and HP groups $(P<0.01$; Fig. 1B), indicating that MALAT1 and its siRNA were successfully transfected into MSCs in the condition of HP..

\section{HP promoted survival and vascularization potential of MSCs in vitro}

HP has been applied to enhance the therapeutic efficacy of MSCs in previous studies. Here, HP mediated effect on MSCs was observed in vitro. It was found that MSCs growth rate was significantly increased in the HP group compared with the normoxia group, and cells apoptosis was decreased $(P<0.01 ;$ Fig. $2 \mathrm{~A}$ and $2 \mathrm{~B})$, The number of vascular branches in the HP group was distinctly higher in contrast to the normoxia group $(P<0.01$, Fig. $2 C$ ). The above mentioned results suggested that HP enhanced survival and vascularization potential of MSCs in vitro.

\section{MALAT1 functioned as a positive modulator in HP mediated MSCs survival and vascularization}

The regulatory role of MALAT1 in HP mediated effects was verified by targeted gene overexpression or knockdown studies. MALAT1 expression status was confirmed by quantitative RT-PCR analysis $(P<0.01$; Fig. 1A and 1B). The HP group showed an upregulated expression of MALAT1 compared with the normoxia group ( $P<0.01$; Fig. 1A and 1B). Transfection of MALAT1 to MSCs further aggrandized cells proliferation rate and reduced apoptosis, whereas silencing of it markedly mitigated cells proliferation and increased apoptosis. The HP + MALAT1 group exhibited a more rapid growth compared with the HP + MALAT1 NC and HP groups $(P<0.01$, Fig. 2A and 2B; $P<0.01)$. However, the HP + si-MALAT1 group presented a much lower growth rate as well as an obvious rise of apoptosis rate in contrast to the $\mathrm{HP}+\mathrm{si}-$ MALAT1 NC and HP groups $(P<0.01$, Fig. $2 A$ and $2 B ; P<0.01)$.

The number of vascular branches in the HP + MALAT1 group was much higher compared with the HP + MALAT1 NC and HP groups ( $P<0.01$, Fig. $2 \mathrm{C}$ ), while the HP + si-MALAT1 group displayed a decreased number of vascular branches in contrast with the HP + si-MALAT1 NC and HP groups ( $<<0.01$, Fig. $2 \mathrm{C}$ ). These indicated that MALAT1 functioned as a positive modulator in HP mediated MSCs survival and vascularization. 


\section{MALAT1 modulated HP induced effects by upregulating the expression of VEGFA}

VEGFA participates in the regulation of MSCs survival and vascularization. In this work, the expression of VEGFA was detected in different experimental groups. It was shown that VEGFA expression was significantly elevated in the HP group $(P<0.01$, Fig. 3$)$. Both the protein and mRNA levels of VEGFA were lifted in the HP + MALAT1 group compared with the HP + MALAT1 NC and HP groups $(P<0.01$, Fig. 3$)$, whereas its expression was decreased in the HP + si-MALAT1 group in contrast with the HP + si-MALAT1 NC and HP groups $(P<0.01$, Fig. 3 ). These results insinuated that MALAT1 could trigger the upregulation of VEGFA, which might contribute to HP mediated MSCs survival and vascularization.

\section{miR-195 negatively regulated VEGFA}

VEGFA has been validated as a target of miR-195 in other previous studies (Fig. 4A) [21]. To figure out whether VEGFA was negatively regulated by miR-195, we inspected its expression level in MSCs treated with miR-195 mimic or miR-195 inhibitor under the environment of HP. Overexpression or inhibition of miR-195 was verified by the detection of its mRNA level ( $P<0.01$, Fig. 4B). It was discovered that both the protein and mRNA levels of VEGFA were obviously downregulated in the HP + miR-195 group compared with the HP + miR-195 NC and HP groups ( $P<0.01$, Fig. 4C and 4D). Nevertheless, there was an observably enriched expression of VEGFA in the HP + miR-195 inhibitor group in contrast with the HP+ miR-195 inhibitor NC and HP groups ( $P<0.01$, Fig. 4C and 4D). All of these demonstrated that miR-195 targeted and negatively regulated VEGFA in MSCs under the condition of HP.

\section{MALAT1 targeted and negatively regulated miR-195}

IncRNAs can act as competing endogenous RNAs (ceRNAs) and suppress microRNAs (miRNAs). They form a regulatory IncRNAs-miRNAs-mRNAs network to mediate the target mRNAs of miRNAs. It has been recorded that MALAT1 is able to function as a molecular sponge of miRNAs to regulate the target mRNA genes. In this study, we concentrated on the interaction between MALAT1 and miR-195. Mutual binding sites of the two noncoding RNAs (ncRNAs) were predicted by the bioinformatics tool (RegRNA2.0, Fig. 5A). Dual luciferase reporter assay was applied to further confirm the interactions between MALAT1 and miR-195. It was indicated that co-transfection of psiCHECK2-MALAT1-WT and miR-195 mimic greatly curtailed the relative luciferase activity $(P<0.01$, Fig. 5B), while there was no alteration of the relative luciferase activity in the psiCHECK2-MALAT1-MU + miR-195 group compared with the psiCHECK2MALAT1-MU + miR-195 NC and blank control groups ( $P<0.01$, Fig. 5B).

The expression of MALAT1 and miR-195 was further checked in the condition of HP. The HP + MALAT1 group presented an obvious decrease of miR-195 compared with the HP + MALAT1 NC and HP groups (P $<0.01$, Fig. 5C). However, the HP + si-MALAT1 group showed a significantly increased expression of miR195 in contrast to the HP + si-MALAT1 NC and HP groups $(P<0.01$, Fig. $5 C)$, The aforementioned results 
ascertained that MALAT1 could competitively inhibit miR-195 to further upregulate the expression of VEGFA.

\section{Discussion}

In this study, we demonstrated that HP promoted MSCs survival and vascularization through the activation of MALAT1/miR-195/VEGFA axis.

MSCs treatment has been developed as an ideal therapeutic strategy for myocardial infarction [1-3]. However, inferior viability of the transplanted stem cells restrains their therapeutic efficacy [4]. HP can effectively enhance the survival of engrafted MSCs and amplify their therapeutic efficiency [6, 7]. HP induces metabolic changes of MSCs and yields higher retention of these cells after transplantation in vivo [8]. Later studies unearth that HP fortifies the survival and proliferation of transplanted MSCs via obstructing signaling molecules of cell death and augmenting angiogenic cytokine secretion [9]. In the current work, it was also revealed that MSCs experienced HP presented a higher proliferation rate and lower apoptosis rate in vitro. In addition, their vascularization potential was also intensified. However, the plausible determinants of HP-mediated effects remained largely unclear.

MALAT1 is a ubiquitously expressed IncRNA with a primary gene sequence of more than $8000 \mathrm{bp}$ [13]. As one of the first identified IncRNAs associated with human disease, it is widely concerned due to its abundant expression and evolutionarily conservation throughout various mammalian species [14, 22]. The cellular and molecular functions of MALAT1 have been extensively studied since its discovery. Accumulating data manifest that MALAT1 exerts critical roles in multifarious biological and pathophysiological processes [23]. Hypoxia directly impacts MALAT1 gene transcription [16, 17]. MALAT1 is recognized as one of the most strongly regulated non-coding transcripts by hypoxia in some cell lines $[17,24]$. MALAT1 can be evoked by hypoxia to dominate cell proliferation and vasculogenesis [25]. Ectopic expression of MALAT1 induced by hypoxia contributes to the regulation of the proliferative phenotype of vascular smooth muscle cells (VSMCs). Evidence has shown that MALAT1 also participates in stem cell biological activities. It is reported that MALAT1 overexpression propels the proliferation of human periodontal ligament stem cell (PDLSC) [26]. MALAT1 has already been identified to be a crucial endogenous regulator in the proliferation, differentiation and angiogenesis properties of MSCs $[15,27,28]$. It facilitates the proliferation, migration, and angiogenesis of MSCs through VEGFA [15]. Knockdown of MALAT1 prevents cell proliferation and angiogenesis of MSCs [15]. In this study, it was found that MALAT1 was significantly increased in MSCs after HP. Overexpression of MALAT1 could further promote MSCs proliferation and reduce cells apoptosis, whereas suppression of its expression impeded cells growth and aggravated apoptosis in the circumstance of HP. These findings suggested that MALAT1 executed a predominant role in HP mediated MSCs survival.

MALAT1 is implicated in endothelial cells (ECs) proliferation, migration and tube formation. It exhibits an enriched expression in vascular cells and displays a pivotal role in modulating sprouting, migration and proliferation of ECs. [29]. Genetic deletion or pharmacological inhibition of MALAT1 impairs vascular 
growth. MALAT1 is uncovered to be one of the most highly expressed IncRNAs in ECs in response to hypoxia [30]. Previous studies reveal that overexpression of MALAT1 in MSCs drives tube formation of HUVECs. MALAT1 knockdown markedly restricts HUVECs angiogenesis and increases vascular permeability [31]. In this work, it was shown that the vasculogenic capacity of MSCs was reinforced after HP. MALAT1 transfection into MSCs further enhanced angiogenic capacity of these cells in HP. However, the number of vascular branches was remarkably declined once MALAT1 was inhibited in MSCs. These implied that HP induced angiogenic potential of MSCs was partially mediated by MALAT1.

As HP promoted survival and vascularization by regulating MALAT1, the downstream molecules were further explored. VEGFA exerts as a crucial mediator of MSCs survival and angiogenesis. Substantial evidences show that upregulation of VEGFA consolidates MSCs survival and their vasculogenic potential $[12,19]$. VEGFA has been acknowledged as a downstream pro-angiogenesis factor of MALAT1[32]. MALAT1 prompts the expression of VEGFA to modulate angiogenesis and epithelial-mesenchymal transition $[33,34]$. It can be recruited to VEGFA pre-mRNA and control the expression of VEGFA isoforms [35]. In this study, it was discovered that both MALAT1 and VEGFA was upregulated in MSCs after HP. Transfection of MALAT1 gave rise to a higher level of VEGFA expression. However, its blockade resulted in an obvious decrease of VEGFA, supporting that MALAT1enhanced MSCs survival and vascularization via upregulating VEGFA in the condition of HP.

microRNAs (miRNAs) are highly conserved non-coding RNAs (ncRNAs) that act as a key orchestrator of numerous biological activities[12]. They take part in the regulation of post-transcriptional gene expression programs by targeting the 3'-UTR of genes. Some miRNAs are corroborated to be tightly linked with MSCs survival and vasculogenesis [12]. Previous studies have notified that miR-195 regulates MSCs proliferation and angiogenesis [21]. Overexpression of miR-195 in human primary MSCs represses cells proliferation rate and diminishes their paracrine effect on angiogenesis [21]. There is another study reported that aberrant expression of miR-195 in old MSCs (OMSCs) provokes stem cell senescence by deteriorating their regenerative capacity, and abolition of miR-195 can reverse stem cell aging [36]. Massive data have sustained that miR-195 mediates cells proliferation and angiogenesis by modulating the exptession of VEGFA [21]. Introduction of exogenous miR-195 results in downregulation of VEGF-A, subsequently leading to cell cycle arrest and exacerbating apoptosis [37]. VEGFA has been authenticated as a direct target of miR-195 and is negatively correlated with miR-195 expression [38, 39]. miR-195 can directly target 3'-UTR of VEGFA and abrogates its expression to further inhibit proliferation and angiogenesis capacities of MSCs [21]. Here, it was also discovered that VEGFA expression was significantly downregulated after the transfection of miR-195 mimic, whereas inhibition of miR-195 conspicuously restored its level, implying that VEGFA was targeted and negatively regulated by miR-195.

The IncRNA-associated competing endogenous RNA (ceRNA) network has attracted much attention recently $[12,32-34]$. Series of records have proved that MALAT1 play a prominent role in cell proliferation, differentiation and angiogenesis by functioning as a ceRNA [40]. One latest study indicates that MALAT1 mediates proliferation and apoptosis of VSMCs by sponging miRNA-124-3p [41]. It has been reported that MALAT1 can act as a sponge of miR-34c and miR-30 to modulate osteogenic 
differentiation of MSCs [42]. Another study shows that MALAT1 promotes the expression of EMT and angiogenesis related genes by sponging miR-126-5p. [43]. Several recent studies have validated MALAT1 as a molecular sponge for miR-195 in some cell lines, including lymphoma and hepatoma cells [44, 45]. However, the relation between MALAT1 and miR-195 in MSCs has not been recorded. In the present work, the posttranscriptional regulation of MALAT1 was explored in MSCs. We proposed that MALAT1 functioned as a sponge for miR-195 to modulate the target gene VEGFA, eventually enhancing MSCs survival and vascularization potential in the condition of HP. Dual luciferase report and qRT-PCR assays were performed to verify the interaction between MALAT1 and miR-195. It was shown that the MALAT1 reporter gene luciferase activity was significantly decreased in miR-195 transfection group in contrast with the blank control and NC groups. qRT-PCR analysis displayed that miR-195 was conversely correlated with MALAT1 expression. Overexpression of MALAT1 significantly decreased miR-195 level in MSCs. However, its depletion caused a pronouncedly increased expression of miR-195. Taken together, these implied that MALAT1 could target and regulate miR-195 as a ceRNA to further upregulate VEGFA.

\section{Conclusion}

In summary, we demonstrated that HP promoted MSCs survival and vascularization through the activation of MALAT1/miR-195/VEGFA axis. We identified a potential ceRNA network by which MALAT1 functioned as a molecular sponge for miR-195 to regulate the expression of VEGFA in HP. Further explorations of these molecules will be conducive for developing new strategies to enhance the therapeutic efficiency of MSCs.

\section{Declarations}

\section{Ethical Approval and Consent to participate}

Animals were obtained from the Animal Experimental Center of the Sun Yat-sen University. All animal handling and procedures were performed in accordance with protocols approved by the Animal Ethics Committee of Sun Yat-sen University (201900005).

\section{Consent for publication}

Not applicable

\section{Availability of supporting data}

All data generated or analyzed during this study are included in this published article.

\section{Competing interests}

The authors declare that they have no competing interests.

\section{Funding}


This study was supported by Grant [2013]163 from Key Laboratory of Malignant Tumor Molecular Mechanism and Translational Medicine of Guangzhou Bureau of Science and Information Technology; Grant KLB09001 from the Key Laboratory of Malignant Tumor Gene Regulation and Target Therapy of Guangdong Higher Education Institutes; National Natural Science Foundation of China (No: 81700242), Science and Technology Foundation of Guangdong Province (No: 2017A020215176), the Medical Science and Technology Research Fund of Guangdong Province (A2017001), the Science and Technology Foundation of Guangzhou City (201704020121) and Yat-Sen Scholarship for Young Scientist.

\section{Authors' contributions}

Jingying Hou conceived the study, participated in the study design and drafted the manuscript; Lingyun Wang and Zhengfei Yang carried out transfection and drafted the manuscript; Guanghui Zheng carried out the molecular assay and cell staining; Tianzhu Guo participated in the cell culture and vector construction; Menglei Yu carried out the statistical analysis. All authors read and approved the final manuscript.

\section{Acknowledgments}

This work was done by the investigators of the Sun Yat-sen Memorial Hospital of Sun Yat-sen University. The authors took responsibility for all aspects of the reliability and had no differences in data presentation and interpretation.

\section{Authors' information}

${ }^{1}$ Guangdong Provincial Key Laboratory of Malignant Tumor Epigenetics and Gene Regulation, the Sun Yat-sen Memorial Hospital of Sun Yat-sen University, 107 Yanjiang Xi Road, Guangzhou, Guangdong, China ${ }^{2}$ Department of Emergency, the Sun Yat-sen Memorial Hospital of Sun Yat-sen University, 107 Yanjiang Xi Road, Guangzhou, Guangdong, China. ${ }^{3}$ Department of Gastroenterology, the Sun Yat-sen Memorial Hospital of Sun Yat-sen University, 107 Yanjiang Xi Road, Guangzhou, Guangdong, China

\section{References}

1. Bagno L, Hatzistergos KE, Balkan W, et al. Mesenchymal stem cell-based therapy for cardiovascular disease: progress and challenges. Mol Ther. 2018; 26(7): 1610-1623.

2. Celikkan FT, Mungan C, Sucu M, et al. Optimizing the transport and storage conditions of current good manufacturing practice -grade human umbilical cord mesenchymal stromal cells for transplantation (HUC-HEART Trial). Cytotherapy. 2019; 21(1): 64-75.

3. Ward MR, Abadeh A, Connelly KA. Concise review: rational use of mesenchymal stem cells in the treatment of ischemic heart disease. Stem Cells Transl Med. 2018; 7(7): 543-550. 
4. De Becker A, Riet IV. Homing and migration of mesenchymal stromal cells: How to improve the efficacy of cell therapy? World J Stem Cells. 2016; 8(3): 73-87.

5. Vu MQ, Der Sarkissian S, Borie M, et al. Optimization of mesenchymal stem cells to increase their therapeutic potential. Methods Mol Biol. 2016; 1416: 275-288.

6. Fujisawa K, Takami T, Okada S, et al Analysis of metabolomic changes in mesenchymal stem cells on treatment with desferrioxamine as a hypoxia mimetic compared with hypoxic conditions. Stem Cells. 2018; 36(8): 1226-1236.

7. Hu Y, Chen $\mathrm{W}, \mathrm{Wu} \mathrm{L}$, et al. Hypoxic preconditioning improves the survival and neural effects of transplanted mesenchymal stem cells via CXCL12/CXCR4 signalling in a rat model of cerebral infarction. Cell Biochem Funct. 2019; 37(7): 504-515.

8. Beegle J, Lakatos K, Kalomoiris S, et al. Hypoxic preconditioning of mesenchymal stromal cells induces metabolic changes, enhances survival, and promotes cell retention in vivo. Stem Cells. 2015; 33(6):1818-1828.

9. Lee JH, Yoon YM, Lee SH, Hypoxic Preconditioning Promotes the Bioactivities of Mesenchymal Stem Cells via the HIF-1 a-GRP78-Akt Axis. Int J Mol Sci. 2017; 18(6): E1320.

10. Hou J, Zhou C, Long $\mathrm{H}$, et al. Long noncoding RNAs: Novel molecules in cardiovascular biology, disease and regeneration. Exp Mol Pathol. 2016; 100(3): 493-501.

11. Hou J, Wang L, Wu Q, et al. Long noncoding RNA H19 upregulates vascular endothelial growth factor A to enhance mesenchymal stem cells survival and angiogenic capacity by inhibiting miR-199a-5p. Stem Cell Res Ther. 2018; 9(1):109.

12. Cui $P$, Zhao $X$, Liu J, et al. miR-146a interacting with IncRNA EPB41L4A-AS1 and IncRNA SNHG7 inhibits proliferation of bone marrow-derived mesenchymal stem cells. J Cell Physiol. 2020; 235(4): 3292-3308.

13. Ji P, Diederichs S, Wang W, et al. MALAT-1, a novel noncoding RNA, and thymosin beta4 predict metastasis and survival in early-stage non-small cell lung cancer. Oncogene. 2003; 22(39): 80318041.

14. Shlyueva D, Stampfel G, and Stark A. Transcriptional enhancers: from properties to genome-wide predictions. Nat Rev Genet.2014; 15: 272-286.

15. Li X, Song Y, Liu F, et al. Long non-coding RNA MALAT1 promotes proliferation, angiogenesis, and immunosuppressive properties of mesenchymal stem cells by inducing VEGF and IDO. J Cell Biochem. 2017; 118(9): 2780-2791.

16. Stone JK, Kim JH, Vukadin L, et al. Hypoxia induces cancer cell-specific chromatin interactions and increases MALAT1 expression in breast cancer cells. J Biol Chem. 2019; 294(29): 11213-11224.

17. Choudhry H, Schödel J, Oikonomopoulos S, et al. Extensive regulation of the non-coding transcriptome by hypoxia: role of HIF in releasing paused RNApol2. EMBO Rep. 2014; 15(1): 70-76.

18. Yoshimoto R, Mayeda A, Yoshida M, et al. MALAT1 long non-coding RNA in cancer. Biochim. Biophys. Acta 2016; 1859: 192-199. 
19. Hou J, Zhong T, Guo T, et al. Apelin promotes mesenchymal stem cells survival and vascularization under hypoxic-ischemic condition in vitro involving the upregulation of vascular endothelial growth factor. Exp Mol Pathol. 2017; 102(2): 203-209.

20. Hou J, Long H, Zhou C, et al. Long noncoding RNA Braveheart promotes cardiogenic differentiation of mesenchymal stem cells in vitro. Stem Cell Res Ther. 2017; 8(1): 4.

21. Almeida CR, Caires H, Pinto MT, et al. miR-195 in human primary mesenchymal stromal/stem cells regulates proliferation, osteogenesis and paracrine effect on angiogenesis. Oncotarget. 2016; 7(1): 722.

22. Zhang $X$, Hamblin MH, Yin KJ. The long noncoding RNA Malat1: Its physiological and pathophysiological functions. RNA Biol. 2017; 14(12): 1705-1714.

23. Lei L, Chen J, Huang J, et al.Functions and regulatory mechanisms of metastasis-associated lung adenocarcinoma transcript 1. J Cell Physiol. 2018; 234(1): 134-151.

24. Voellenkle C, Garcia-Manteiga JM, Pedrotti S, et al. Implication of Long noncoding RNAs in the endothelial cell response to hypoxia revealed by RNA-sequencing. Sci Rep. 2016; 6: 24141.

25. Brock $M$, Schuoler $C$, Leuenberger $C$, et al. Analysis of hypoxia-induced noncoding RNAs reveals metastasis-associated lung adenocarcinoma transcript 1 as an important regulator of vascular smooth muscle cell proliferation. Exp Biol Med (Maywood). 2017; 242(5): 487-496.

26. Chen $P 1$, Huang $Y$, Wang $Y$, et al. MALAT1 overexpression promotes the proliferation of human periodontal ligament stem cells by upregulating fibroblast growth factor 2. Exp Ther Med. 2019; 18(3): 1627-1632.

27. Tan YF, Tang L, OuYang WX, et al. $\beta$-catenin-coordinated IncRNA MALAT1 up-regulation of ZEB-1 could enhance the telomerase activity in HGF-mediated differentiation of bone marrow mesenchymal stem cells into hepatocytes. Pathol. Res Pract. 2019; 215(3): 546-554.

28. Yi J, Liu D, Xiao J. LncRNA MALAT1 sponges miR-30 to promote osteoblast differentiation of adipose-derived mesenchymal stem cells by promotion of Runx2 expression. Cell Tissue Res. 2019; 376(1): 113-121.

29. Michalik KM, You X, Manavski Y, et al. Long noncoding RNA MALAT1 regulates endothelial cell function and vessel growth. Circ Res 2014; 114: 1389-1397.

30. Moreau PR, Örd T, Downes NL, et al. Transcriptional Profiling of Hypoxia-Regulated Non-coding RNAs in Human Primary Endothelial Cells. Front Cardiovasc Med. 2018; 5: 159.

31. Li Y, Wu Z, Yuan J, et al. Long non-coding RNA MALAT1 promotes gastric cancer tumorigenicity and metastasis by regulating vasculogenic mimicry and angiogenesis. Cancer Lett. 2017; 395: 31-44.

32. Ren L, Wei C, Li K, et al. LncRNA MALAT1 up-regulates VEGF-A and ANGPT2 to promote angiogenesis in brain microvascular endothelial cells against oxygen-glucose deprivation via targetting miR-145. Biosci Rep. 2019; 39(3): BSR20180226.

33. Sun Z, Ou C, Liu J, et al, YAP1-induced MALAT1 promotes epithelial-mesenchymal transition and angiogenesis by sponging miR-126-5p in colorectal cancer. Oncogene. 2019; 38(14): 2627-2644. 
34. Han N, Tian $\mathrm{W}, \mathrm{Yu} \mathrm{N}$, et al. YAP1 is required for the angiogenesis in retinal microvascular endothelial cells via the inhibition of MALAT1-mediated miR-200b-3p in high glucose-induced diabetic retinopathy. J Cell Physiol. 2020; 235(2): 1309-1320.

35. Pruszko M, Milano E, Forcato $M$, et al.The mutant p53-ID4 complex controls VEGFA isoforms by recruiting IncRNA MALAT1. EMBO Rep. 2017; 18(8): 1331-1351.

36. Okada M, Kim HW, Matsu-ura K, et al. Abrogation of age-induced microrna-195 rejuvenates the senescent mesenchymal stem cells by reactivating telomerase.abrogation of age-induced microrna195 rejuvenates the senescent mesenchymal stem cells by reactivating telomerase. Stem Cells. 2016; 34(1): 148-159.

37. Maroof H, Irani S, Arianna A, et al. Interactions of vascular endothelial growth factor and p53 with mir-195 in thyroid carcinoma: possible therapeutic targets in aggressive thyroid cancers. Curr Cancer Drug Targets. 2019; 19(7): 561-570.

38. Zhao WJ, Zhang HF, Su JY. Downregulation of microRNA-195 promotes angiogenesis induced by cerebral infarction via targeting VEGFA Mol Med Rep. 2017; 16(4): 5434-5440.

39. Liu H, Chen Y, Li Y, et al. miR-195 suppresses metastasis and angiogenesis of squamous cell lung cancer by inhibiting the expression of VEGF. Mol Med Rep. 2019; 20(3): 2625-2632.

40. Hong S, Hu S, Kang Z, et al. Identification of functional IncRNAs based on competing endogenous RNA network in osteoblast differentiation. J Cell Physiol. 2020; 235(3): 2232-2244.

41. Cheng $C, X u$ BL, Sheng JL, et al. LncRNA MALAT1 regulates proliferation and apoptosis of vascular smooth muscle cells by targeting miRNA-124-3p/PPARa axis. Eur Rev Med Pharmacol Sci. 2019; 23(20):9025-9032.

42. Yang $X$, Yang J, Lei P, et al. LncRNA MALAT1 shuttled by bone marrow-derived mesenchymal stem cells-secreted exosomes alleviates osteoporosis through mediating microRNA-34c/SATB2 axis. Aging (Albany NY). 2019; 11(20): 8777-8791.

43. Sun Z, Ou C, Liu J, et al. YAP1-induced MALAT1 promotes epithelial-mesenchymal transition and angiogenesis by sponging miR-126-5p in colorectal cancer. Oncogene. 2019; 38(14): 2627-2644.

44. Wang QM, Lian GY, Song Y, et al. LncRNA MALAT1 promotes tumorigenesis and immune escape of diffuse large B cell lymphoma by sponging miR-195. Life Sci. 2019; 231: 116335.

45. Liu D, Zhu Y, Pang J, et al. Knockdown of long non-coding RNA MALAT1 inhibits growth and motility of human hepatoma cells via modulation of miR-195. J Cell Biochem. 2018; 119(2): 1368-1380.

\section{Figures}


Fig 1

A

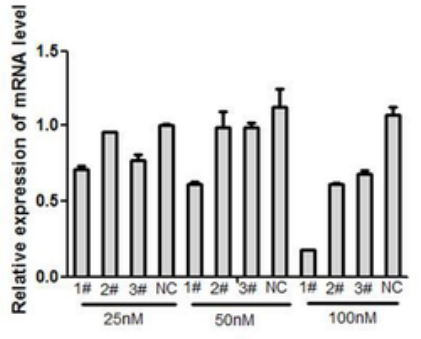

B

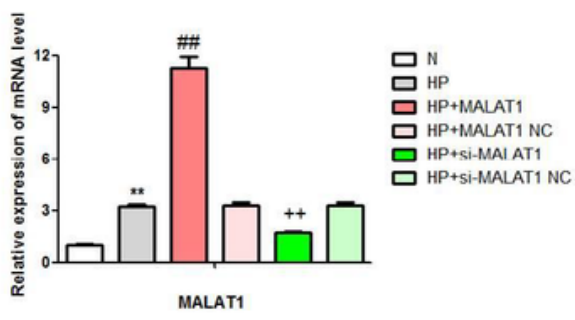

\section{Figure 1}

Interference efficiency of MALAT1 siRNA and the expression of MALAT1 in different groups. (A) For the evaluation of interference efficiency of MALAT1 siRNA, mRNA expression level of MALAT1 was examined by using qRT-PCR. 1\#, 2\# and 3\# represented MALAT1-siRNA1\#, MALAT1-siRNA2\# and MALAT1siRNA3\# respectively. The transfection concentration of the siRNA interference sequences were designed as 25nM, 50nM, and 100nM respectively. (B) Detection of the expression of MALAT1 in different groups. 
N: normoxia; HP: hypoxia preconditioning; HP + MALAT1: MSCs transfected with MALAT1 and experienced HP; MSCs + MALAT1 NC: MSCs transfected with MALAT1 scramble RNA and experienced HP; HP + si-MALAT1: MSCs transfected with MALAT1 siRNA and experienced HP; HP + si-MALAT1 NC: MSCs transfected with MALAT1 siRNA scramble and experienced HP. ${ }^{* * P}<0.01$ vs N囚\#\# $P<0.01$ vs HP and HP + MALAT1 NC, ++ P<0.01 vs HP and HP + si-MALAT1 NC.
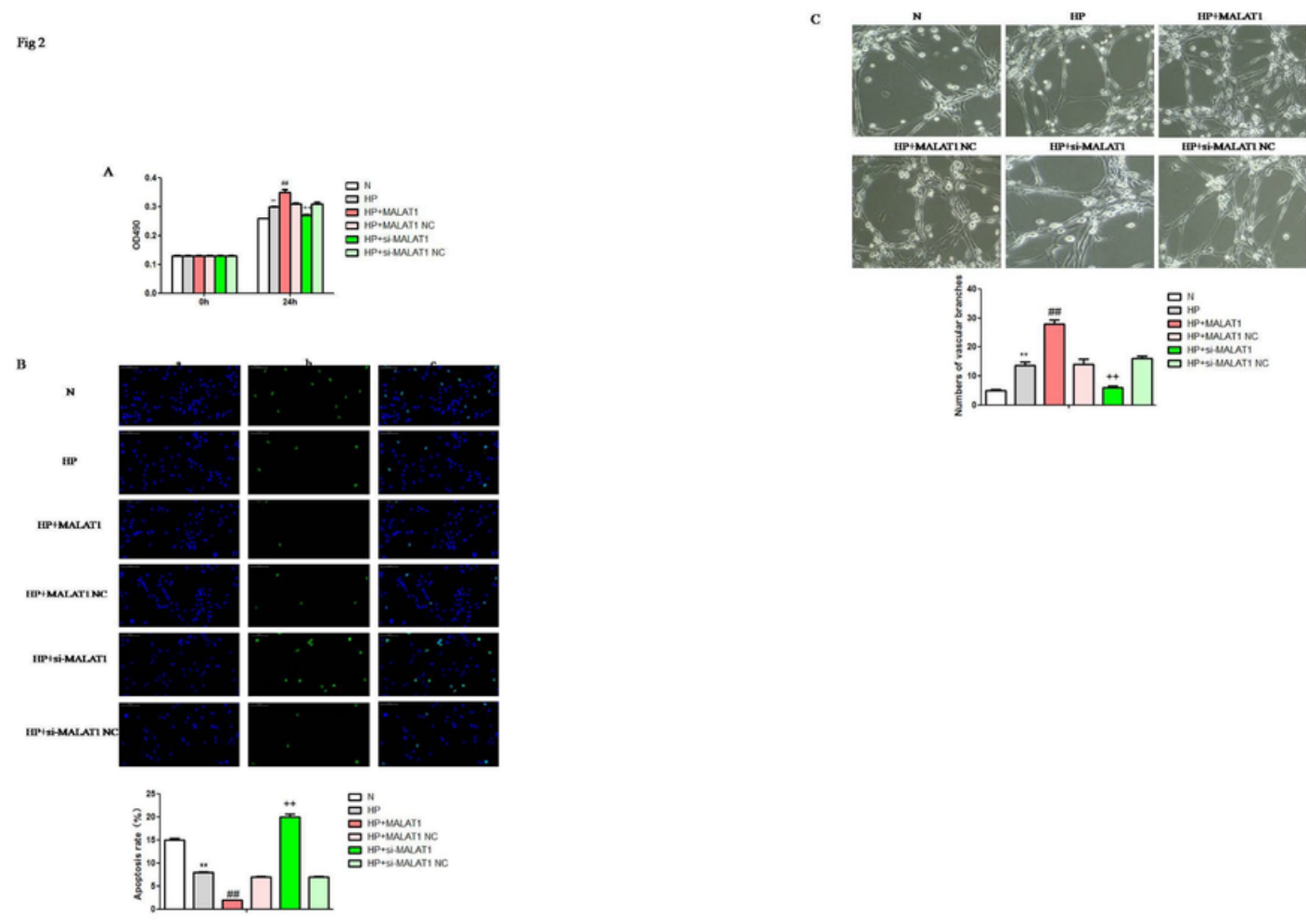

\section{Figure 2}

MALAT1 functioned as a positive modulator in HP mediated MSCs survival and vascularization. (A) Showed the proliferation of MSCs. Cells proliferation of different groups was detected by MTS assay and evaluated by optical density (OD) value. (B) Showed the TUNEL staining of the apoptosis of MSCs and the comparison of apoptosis rates in different groups. a: DAPI staining localization, b: TUNEL staining, c: overlap figure of $a$ and $b$. (C) Showed the results of tube formation assay in different groups. N: normoxia; HP: hypoxia preconditioning; HP + MALAT1: MSCs transfected with MALAT1 and experienced HP; MSCs + MALAT1 NC: MSCs transfected with MALAT1 scramble RNA and experienced HP; HP + siMALAT1: MSCs transfected with MALAT1 siRNA and experienced HP; HP + si-MALAT1 NC: MSCs 
transfected with MALAT1 siRNA scramble and experienced HP. ** $P<0.01$ vs N囚\#\# $\mathrm{P}<0.01$ vs HP and HP + MALAT1 NC, ++ P<0.01 vs HP and HP + si-MALAT1 NC.

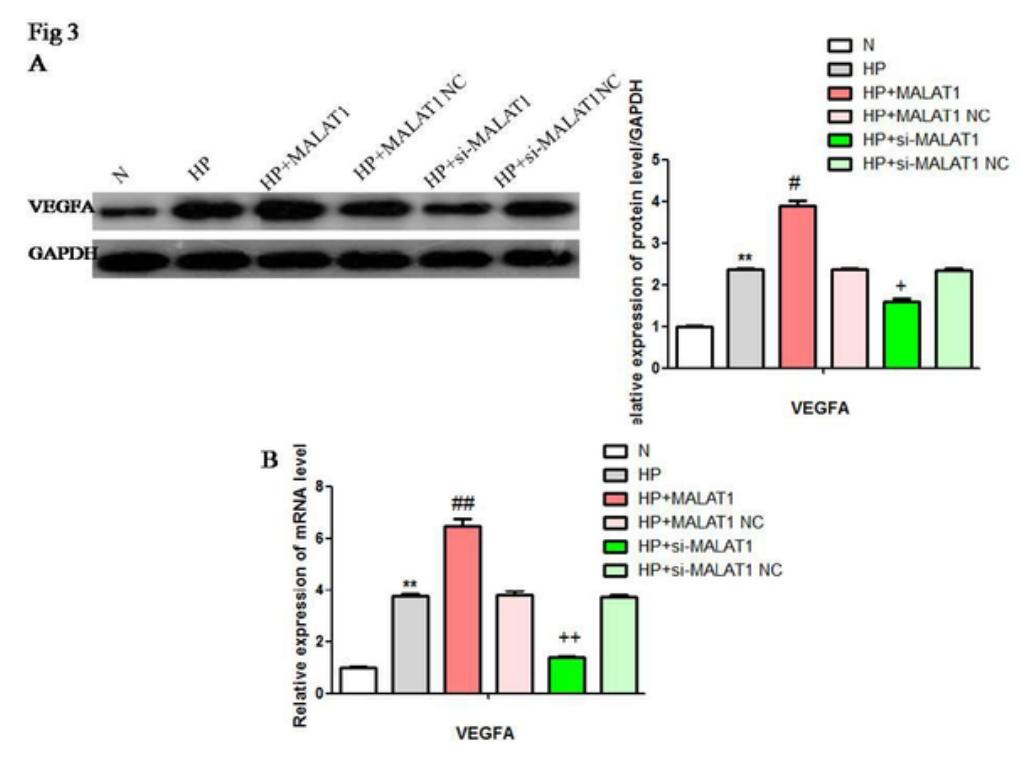

Figure 3

MALAT1 upregulated the expression of VEGFA in HP The expression of VEGFA was detected in different experimental groups. (A) Westernblot analysis of the protein level of VEGFA. (B) qRT-PCR analysis of the mRNA level of VEGFA. N: normoxia; HP: hypoxia preconditioning; HP + MALAT1: MSCs transfected with 
MALAT1 and experienced HP; MSCs + MALAT1 NC: MSCs transfected with MALAT1 scramble RNA and experienced HP; HP + si-MALAT1: MSCs transfected with MALAT1 siRNA and experienced HP; HP + siMALAT1 NC: MSCs transfected with MALAT1 siRNA scramble and experienced HP. **P<0.01 vs N, \#\# $\mathrm{P}<0.01$ vs HP and HP + MALAT1 NC, ++ P<0.01 vs HP and HP + si-MALAT1 NC, \# P<0.05 vs HP and HP + MALAT1 NC, + P<0.05 vs HP and HP + si-MALAT1 NC.

Fig 4
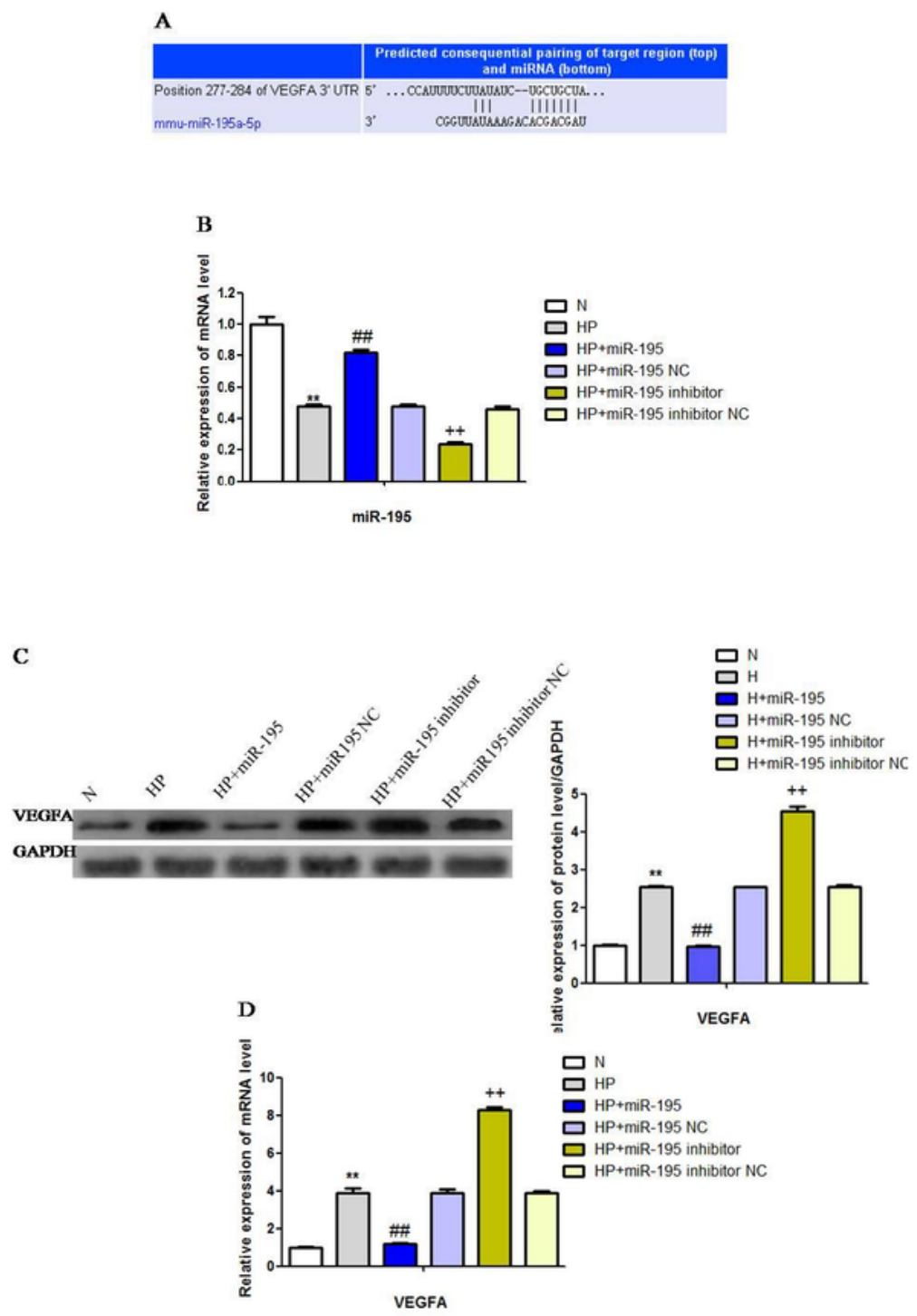

Figure 4 
miR-195 downregulated the expression of VEGFA in HP The expression of VEGFA was detected in different groups. (A) Potenital binding site of VEGFA and miR-195. (B) The expression of miR-195 was analyzed by qRT-PCR. (C) Westernblot analysis of the protein level of VEGFA. (D) qRT-PCR analysis of the mRNA level of VEGFA. N: normoxia; HP: hypoxia preconditioning; HP+miR-195: MSCs transfected with miR-195 mimic and experienced HP; MSCs+ miR-195 NC: MSCs transfected with miR-195 mimic negative control and experienced HP; HP+ miR-195 inhibitor: MSCs transfected with miR-195 inhibitor and experienced HP; HP+ miR-195 inhibitor NC: MSCs transfected with miR-195 inhibitor negative control and experienced HP. ${ }^{*} P<0.01$ vs $\mathrm{N} \rrbracket \# \# P<0.01$ vs HP and HP + miR-195 NC, ++ $\mathrm{P}<0.01$ vs HP and HP + miR195 inhibitor NC. 

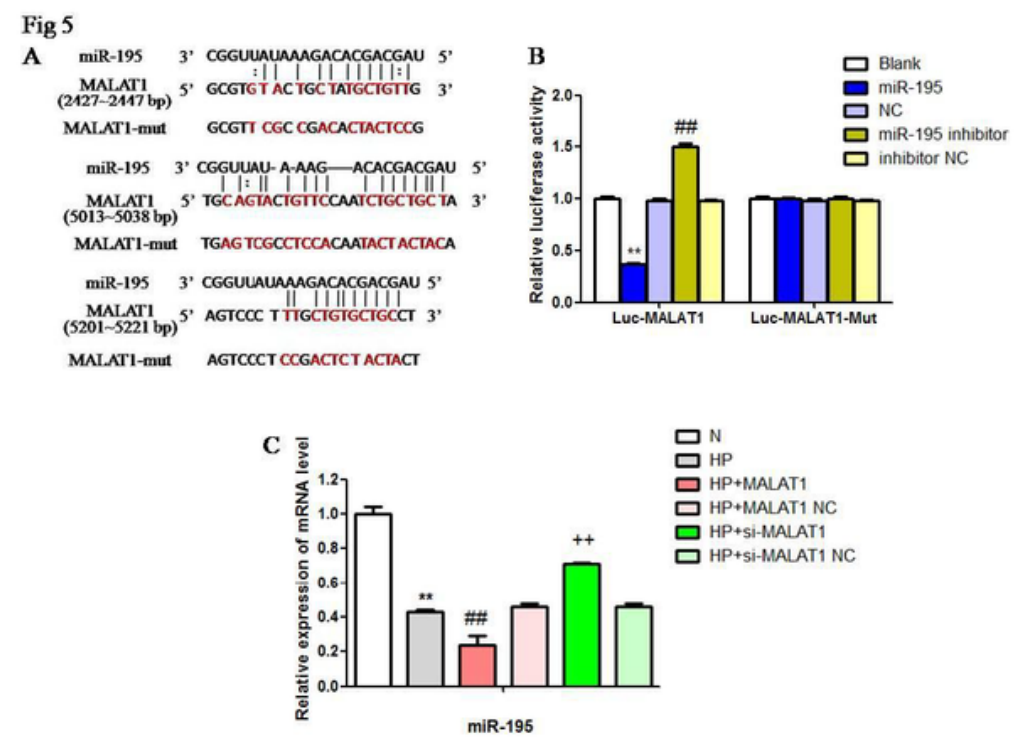

\section{Figure 5}

MALAT1 targeted and negatively regulated miR-195 (A) Potential binding sites of MALAT1 and miR-195. Relevant mutation sites was shown in red. (B) The result of promega dual-luciferase reporter gene assay. Cells were transiently transfected with a luciferase reporter contained the wild type (MALAT1-WT) or mutant MALAT1 (MALAT1-MU). Measurement was performed with the dual-luciferase reporter assay system, and luciferase activity was analysed. Blank control: MSCs were transfected with a luciferase 
reporter contained the MALAT1-WT or MALAT1-MU; miR-195: MSCs were co-transfected with miR-195 mimic and a luciferase reporter contained the MALAT1-WT or MALAT1-MU; miR-195 NC: MSCs were cotransfected with miR-195 mimic negative control and a luciferase reporter contained the MALAT1-WT or MALAT1-MU; miR-195 inhibitor: MSCs were co-transfected with miR-195 inhibitor and a luciferase reporter contained the MALAT1-WT or MALAT1-MU; miR-195 inhibitor NC: MSCs were co-transfected with miR-195 inhibitor negative control and a luciferase reporter contained the MALAT1-WT or MALAT1-MU. (C) Represented the alteration of miR-195 expression level after MALAT1 transfection or knockdown. qRTPCR were performed to explore the expression of miR-195 in different groups. N: normoxia; HP: hypoxia preconditioning; HP + MALAT1: MSCs transfected with MALAT1 and experienced HP; MSCs + MALAT1 NC: MSCs transfected with MALAT1 scramble RNA and experienced HP; HP + si-MALAT1: MSCs transfected with MALAT1 siRNA and experienced HP; HP + si-MALAT1 NC: MSCs transfected with MALAT1 siRNA scramble and experienced HP. ${ }^{* * P}<0.01$ vs N囚\#\# P<0.01 vs HP and HP + MALAT1 NC, ++ $P<0.01$ vs HP and HP + si-MALAT1 NC. 